\title{
Modification of arc emitted W-particles in a model scrape-off layer plasma
}

\author{
M Laux ${ }^{2}$, M Balden ${ }^{1}$ and P Siemroth ${ }^{3}$ \\ ${ }^{1}$ Max-Planck-Institut für Plasmaphysik,EURATOM Association, Boltzmannstr.2, D-85748 Garching \\ ${ }^{2}$ Teilinstitut Greifswald, Wendelsteinstr.1, D-17491 Greifswald, Germany \\ ${ }^{3}$ Arc Precision Sources, Coatings and Analysis GmbH, Bahnhofstr.1, D-15745, Wildau, Germany \\ E-mail: michael.laux@ipp.mpg.de
}

\begin{abstract}
In fusion machines (like e.g. ASDEX Upgrade and JET) equipped with W-coated plasma facing components (PFCs) arc tracks are observed post-mortem and W-particles are identified as a component of the dust.

Results from laboratory arcing of W-coated PFC-material concerning the size, velocity, and direction of macro-particles are combined with model calculations of the heating, cooling, and evaporation of $\mathrm{W}$ particles flying through a simplified scrape-off layer (SOL) plasma to assess the role of arc produced particles as a source of W-impurities. W atoms or ions eroded from a macro-particle flying across the SOL plasma are not subject to prompt re-deposition onto a PFC. Therefore, macro-particles constitute an essential source of $\mathrm{W}$ in the SOL and even may result in an impurity input into the region of confined plasma if the particle succeeds to path the SOL and cross the last closed flux surface (LCFS).

The main result of the modelling is that a W-particle from the large-size end of the distribution obtained in laboratory arcing and having a typical velocity (also known from laboratory experiment) is able to pass a SOL of $10 \mathrm{~cm}$ thickness filled with a realistic plasma and, finally, inject a certain amount of Wmaterial into the confined plasma region.
\end{abstract}

Keywords arcing, Plasma Facing Components, Tungsten, particles, dust, Scrape-Off Layer

\section{Introduction}

Arcing is one process of plasma-surface interaction (PSI) occurring on PFCs of fusion devices. If arcs are burning at the first wall liquid particles as a typical product of arc erosion will be ejected from the typical craters (figure 1) into the SOL [1, 2, 3, 4, 5]. During its flight through the plasma the particles will be evaporated $[6,7]$ introducing wall material into the SOL and, eventually, some may even cross the LCFS thereby injecting a fraction of the impurity material into the confined central plasma column. If W was chosen as the principal PFC material (as in ASDEX Upgrade and JET realized and for ITER planned) any erosion resulting in macro-particle emission is important because it potentially bypasses the desired prompt re-deposition of $\mathrm{W}$ [8].

To assess the role of those particles as a source of $\mathrm{W}$-impurities model calculations of the heating, cooling, and evaporation of a W particle flying through the SOL plasma have to be combined with information about the size, velocity, and direction of macro-particles ejected from an arc. Fortunately, this information can be obtained from laboratory experiments with vacuum arcs on the relevant Wcoated PFC-material because the cathode plasma of an arc dominates the embedding SOL-plasma completely [9]. The pressure of the cold and very dense cathode plasma $\left(\mathrm{k}_{\mathrm{B}} \mathrm{T}_{\mathrm{e}}=1 \ldots 2 \mathrm{eV}, \mathrm{n}=10^{24} \ldots 10^{26} \mathrm{~m}\right.$ ${ }^{3}$ ) produced by local explosive evaporation of solid material is typically 4 to 5 orders of magnitude larger than that of a typical SOL-plasma. Therefore, any pre-existing plasma in front of the developing cathode spot will be expelled completely by the cathodic plasma cloud and, as a consequence, crater formation and particle ejection are in very strong analogy to the case of a vacuum arc (as used in the laboratory experiments).

\section{Laboratory experiments}

\subsection{The experimental set-up}

The experiments were carried out using an all metal 4-way cross as laboratory vacuum chamber having one pumping port, two ports for target holder, current feedthroughs, and ignition tip, and a port with a large window for optical observation of the cathode with cameras (see figure 2). Two large coils are situated to the left and the right of the cross producing a constant magnetic field ( $\leq 140 \mathrm{mT}$ ) parallel to the front of the cathode. After baking to $150^{\circ} \mathrm{C} \ldots 250^{\circ} \mathrm{C}$ a typical base pressure of $1.5 \times 10^{-7} \mathrm{~Pa}$ was reached. The discharge was ignited by a $10 \mathrm{kV}$ pulse applied to a dedicated electrode (a $300 \mu \mathrm{m} \mathrm{W}$-wire 
wound around a ceramics stick of $3 \mathrm{~mm} \varnothing$ in touch with the cathode surface) whereas the chamber was acting as anode. The discharge duration varied from $300 \mu$ s to $5 \mathrm{~ms}$. The arcs were ignited and burned on realistic W-coated graphite PFCs. Samples $\left(40 \mathrm{~mm} \times 30 \mathrm{~mm} \times 16 \mathrm{~mm}\right.$, graphite, $\rho \approx 1.88 \mathrm{~g} / \mathrm{cm}^{3}$, coated by $5 \mu \mathrm{m} \mathrm{W}$ ) originally produced for the usage in AUG have been mounted as the cathode. Two different cameras (a Phantom v7.3, monochrome high-speed video, and an EX-F1, Casio Exilim in video-mode) took pictures through the observation port. That way optical imaging of the spot on the cathode surface as well as of droplets emitted from the spot has been achieved. Both cameras were oriented almost perpendicular (axis $<10^{\circ}$ to the surface normal) to the sample surface. The resulting usable resolution was $\approx 160 \mu \mathrm{m}$. Particle velocities are deduced from observations of the arc discharge with the fast camera. For the monitoring of their tracks a set up with a side view on the sample was used preferentially. Here the very luminous arc spots did not dominate the picture and, therefore, long tracks of flying particles could be recorded even if they were dim.

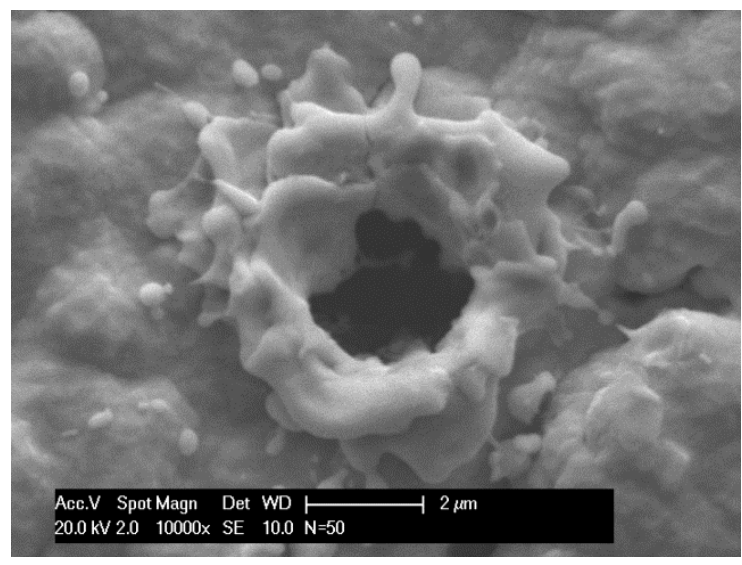

Figure 1. Typical arc crater on the W-coated graphite cathode showing remnants of droplets.

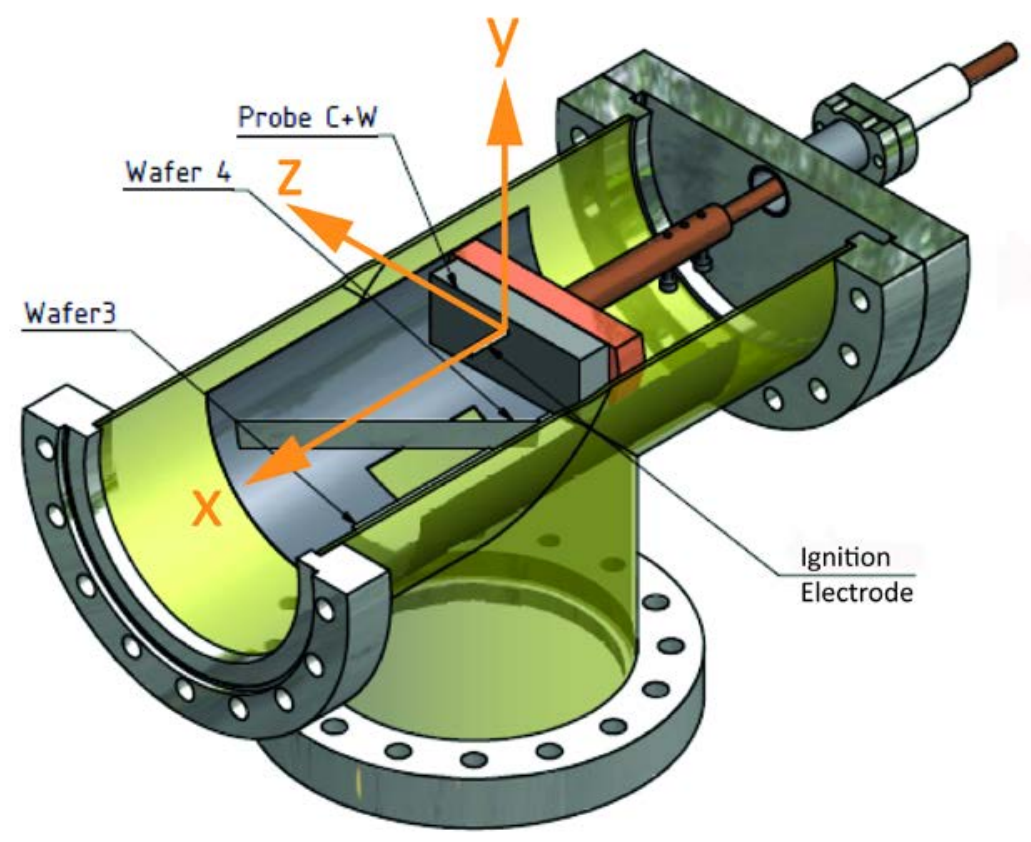

Figure 2. Horizontal cross section through the arcing chamber showing the cathode, the ignition pin, the Si-wafers, and the coordinate system.

Inside the chamber exchangeable Si-wafers were mounted as collector plates (compare figure 2) to catch macro-particles emitted by the burning arc. The collected particles are analysed in selected areas on the wafer to reveal their size, shape, and chemical composition. Combining the geometry of the experimental set-up and the locations of investigated areas on the wafer the directions of the particle orbits are reconstructed. 
Piecewise tracks of flying particles emitted from arc-spots have been observed from high-speed videos. As a result the velocity distribution of particles was constructed having two distinct contributions (one at $\langle\mathrm{v}\rangle \approx 21 \mathrm{~m} / \mathrm{s}, \sigma_{v} \approx 11 \mathrm{~m} / \mathrm{s}$, and the other at $\langle\mathrm{v}\rangle \approx 77 \mathrm{~m} / \mathrm{s}, \sigma_{\mathrm{v}} \approx 23 \mathrm{~m} / \mathrm{s}$ ) (see figure 6 in [1]). Applying a dedicated automated program SEM-images taken from the Si-wafers could be inspected for particles. As the result coordinates, elemental composition, and characteristics of the shape of a large number of particles could be obtained. Fig. 3 shows the diameter distribution of particles containing more than 90 at\% $\mathrm{C}$ and those with a detectable $\mathrm{W}$-content lacking other impurities $\left(\mathrm{C}_{\mathrm{C}}+\mathrm{C}_{\mathrm{W}}>90 \%\right)$, respectively.

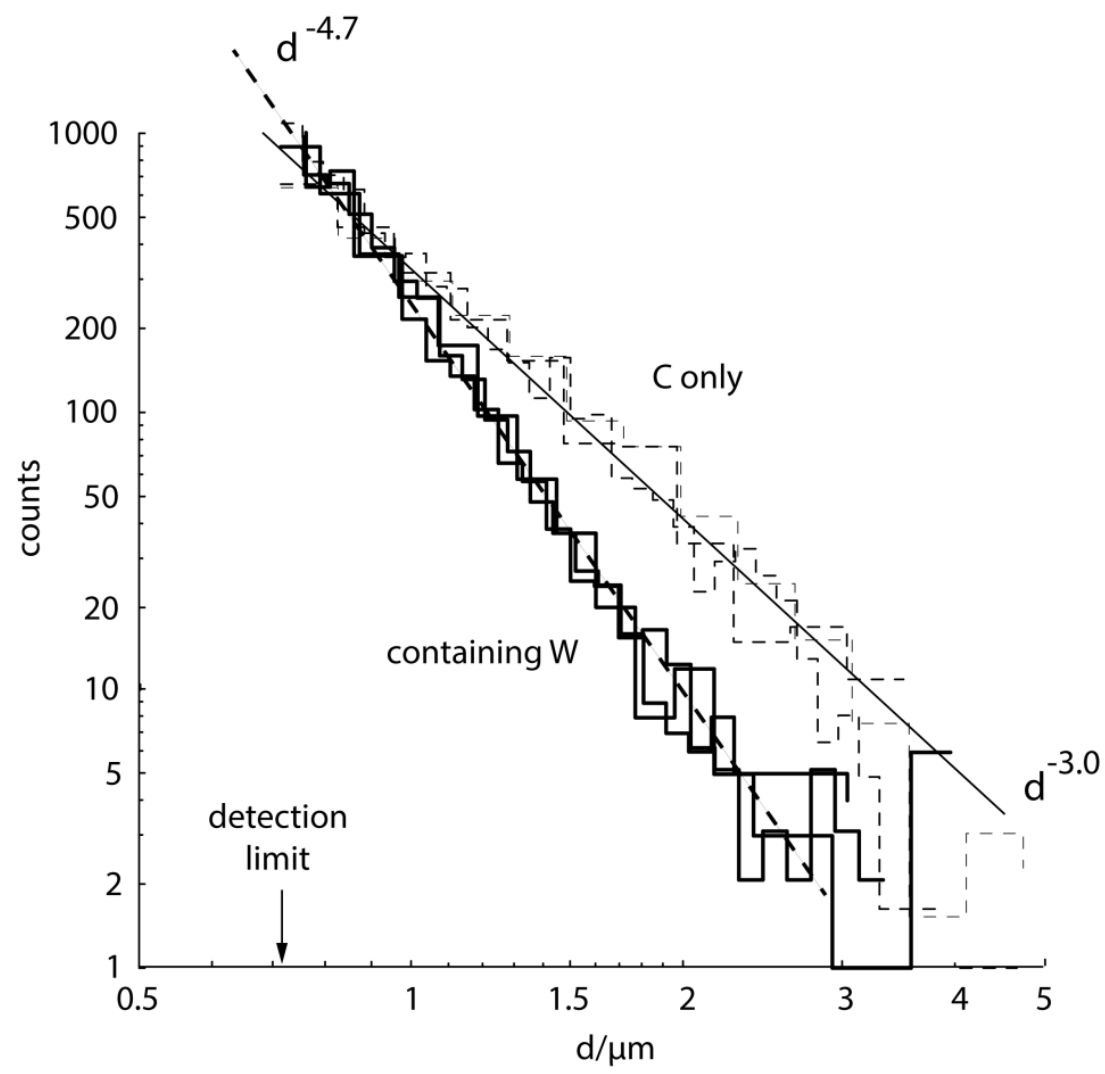

Figure 3. number of particles having diameters within a given interval selected for the elemental composition ( $>90$ at $\% \mathrm{C}, \mathrm{c}_{\mathrm{C}}+\mathrm{c}_{\mathrm{W}}>90$ at $\%$ ). The actual automated particle recognition is limited to $\mathrm{d}>0.7 \mu \mathrm{m}$.

For $\mathrm{C}$ the distribution follows approximately a power law (exponent $\approx-3$ ) whereas for $\mathrm{W}$ containing particles it is much steeper $(\approx-4.7)$. Generally the number of $\mathrm{W}$ containing particles is much smaller than the number of $\mathrm{C}$ particles.

Localized (essentially rectangular) regions distributed over the Si-wafers have been analyzed to obtain distribution at different angles. The individual location was represented by the center of the rectangle and a coordinate system at the ignition location of the arcs was introduced (see figure 2). Hence, the distance of the analyzed region from the emitting arc-spot on the cathode and the angle of the paths of the arriving particles with respect to the cathode surface could be calculated and, therefore, distributions of particle sizes for dedicated ejection angles can be given. Here it is assumed that (i) during its lifetime the arc-spot stays close to the ignition point and (ii) that the sticking of the particles impinging on the wafers is complete. To select the particle classes of interest from the database produced by the automated SEM-program the limits for the elemental composition and the tolerated deviation from an ideal sphere have to be presumed. The measured areal densities of particles of a given diameter are corrected for the distance of the location from the cathode spot and for the inclination of the wafer's surface (e.g. a projection onto the unit-sphere was made).

As the result (given in figure 4 for all particles fulfilling $\mathrm{C}_{\mathrm{C}}+\mathrm{C}_{\mathrm{W}}>90$ at\%) a complicated dependence on the angle of ejection was observed. Nevertheless it can be stated that large particles ( $d>2 \mu \mathrm{m})$ are preferentially emitted under shallow angles with the cathode surface $\left(80^{\circ} \ldots 90^{\circ}\right.$ with the normal). The largest particles found have diameters $\approx 10 \mu \mathrm{m}$ for $\mathrm{W}$ as well as $\mathrm{C}$. Smaller objects $(\mathrm{d}<2 \mu \mathrm{m})$ contribute a larger amount at more elevated angles with the surface $\left(40^{\circ} \ldots 50^{\circ}\right.$ with the normal). 


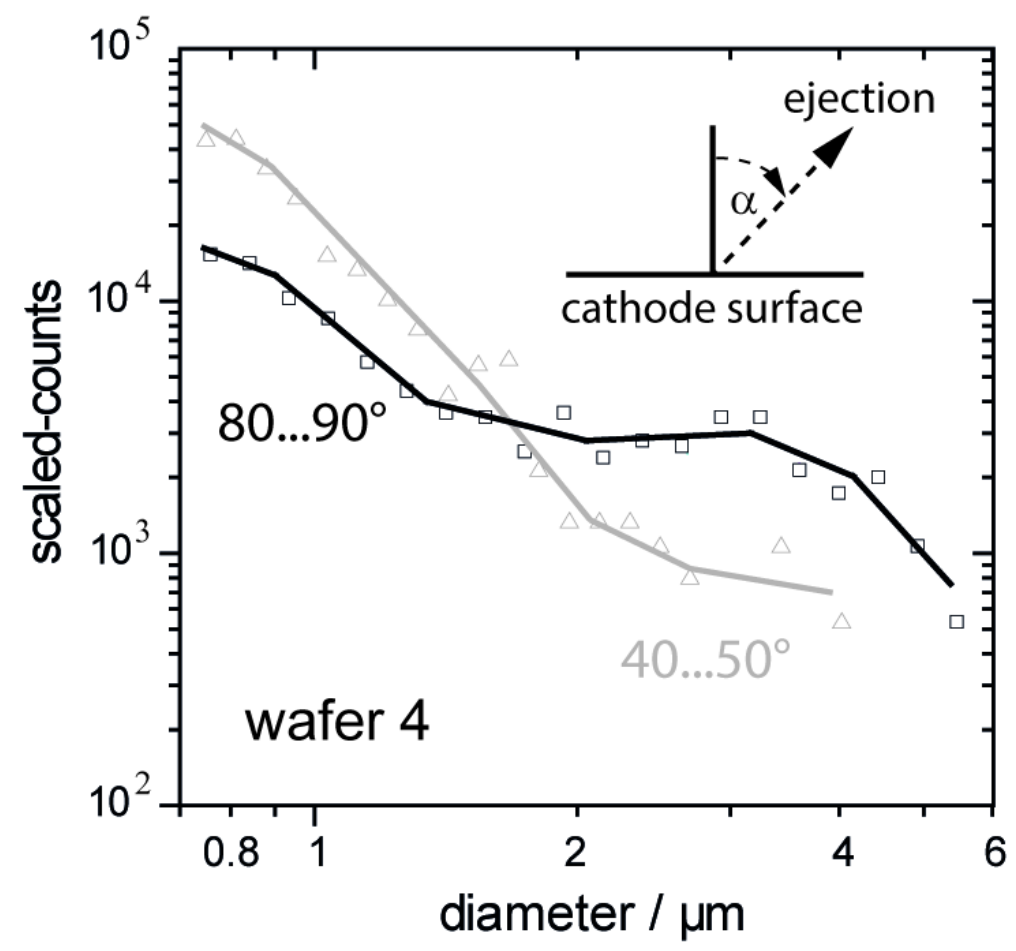

Figure 4. diameter distributions for different angles between the particles path and the normal of the cathode surface (particles with $\mathrm{c}_{\mathrm{C}}+\mathrm{C}_{\mathrm{W}}>90$ at $\%$ only).

\section{Modeling of the particles flight}

\subsection{The SOL}

A very simple slab SOL is assumed (figure 5) framed by the plane wall on one side and a plane LCFS on the opposite side. A strong magnetic field B (typically several T) exists and is oriented parallel to the surface of the wall. As a consequence the SOL plasma is described by one-dimensional profiles of the essential parameters density $n$ and electron temperature $T_{e}$ along the perpendicular coordinate $x$ (originating at the wall). The profiles are approximated by two exponential decays characterized by the respective values $n$ (LCFS) and $k_{B} T_{e}$ (LCFS) at the LCFS and the related decay lengths $\lambda_{n}$ and $\lambda_{\text {Te }}$, respectively. The thickness of the SOL is given by $d_{\mathrm{SOL}}$. Any particle emitted along a track not parallel to the $\mathrm{x}$-axis is described by a projected velocity $\mathrm{v}_{0, \perp}=\mathrm{v}_{0} \cos (\alpha)$, where $\alpha$ is the angle between the track and the $\mathrm{x}$-axis.

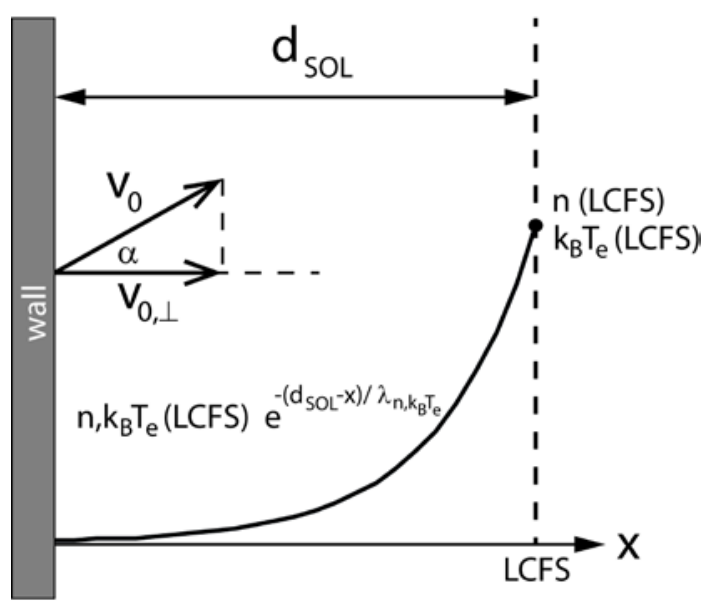

Figure 5. scheme of the SOL and the density and temperature profiles 


\subsection{The particle}

The particle is assumed to consist of tungsten only having a spherical geometry with the diameter $\mathrm{d}$ that may vary during the flight through the SOL. Using the density $\rho$ of the particle's material the diameter can be related to the mass $m$. Knowing the actual diameter the volume $V_{p}$, the surface $O_{p}$, and the projected surface $\mathrm{O}_{\perp}=2 \times \pi / 4 \times \mathrm{d}^{2}$ of the sphere can be calculated. The specific heat capacity $\mathrm{c}_{\mathrm{W}}$ of $\mathrm{W}$ describes the thermal heat content of the particle and is approximated by the value $\mathrm{c}_{\mathrm{W}} \approx 200 \mathrm{~J} /(\mathrm{kg} \times \mathrm{K})$ that is valid for temperatures from $2000 \mathrm{~K}$ on. A possible temperature dependence of the density is neglected as well as the thermal expansion of the particle.

In principle the starting temperature $\mathrm{T}_{0}$ of the particle is a free parameter, but in the runs described below it has been assumed that $T_{0}=T_{m}=3395 K$, the melting temperature of $\mathrm{W}$, because the particles originate from the molten $\mathrm{W}$ pool in the cathode spot. The initial velocity $\mathrm{v}_{0}$ is also a free parameter but is found in the laboratory experiments to be typically distributed $\left(\sigma_{\mathrm{v}}=11 \mathrm{~m} / \mathrm{s}\right)$ around $20 \mathrm{~m} / \mathrm{s}$ $\left(\left\langle\mathrm{v}_{0}\right\rangle=21 \mathrm{~m} / \mathrm{s}\right)$. This is a value valid for particles of almost pure W composition. Particles with a large Ccontent have typically higher velocities.

The particle is assumed to be homogeneous as far as density and temperature are concerned and to stay spherical even if its mass is changing. It moves as a rigid body with no additional internal degrees of freedom (e.g. rotation, oscillations etc.).

\subsection{The heating}

The heat flux density $p_{\text {rad }}$ due to the radiation from the particle's surface is given by $p_{\mathrm{rad}}=-\sigma \times \mathrm{T}^{4}$, with $\sigma=5.67 \times 10^{-8} \mathrm{~W} /\left(\mathrm{m}^{2} \mathrm{~K}^{4}\right)$. Of course the particle also receives thermal radiation from the wall as well as from the main plasma. The radiated power will be

$P_{\text {rad }}=-O_{p} \times \sigma\left(T^{4}-T_{s}^{4}\right)$

where $T$ is the temperature of the particle and $T_{s}$ an effective temperature of the surroundings. The cooling of the particle by evaporation is related to the accompanying mass loss via the specific evaporation heat $\mathrm{q}_{\mathrm{ev}} \approx 4350 \mathrm{~kJ} / \mathrm{kg}$ for $\mathrm{W}$. This mass loss is given by the evaporation rate $\mathrm{r}(\mathrm{T})$ as a function of the temperature of the particle. The rate $\mathrm{r}$ can be approximated by the expression [10]

$r(T)=r\left(T_{m}\right) \times \exp \left[\beta\left(T-T_{m}\right)+\delta\left(T-T_{m}\right)^{3}\right]$

with $\beta=7.5029 \times 10^{-3} / \mathrm{K}$ and $\delta=1.8944 \times 10^{-9} / \mathrm{K}^{3}$ for $\mathrm{W}$. As a result the heat flux due to evaporation is

$P_{e v}=-O_{p} \times r(T) \times q_{e v}$

The major heating of the particle is by its convective contact to the imbedding SOL plasma given by

$p_{c}=\gamma \times k_{B} T_{e} \times \Gamma=\gamma /{ }_{4} \times k_{B} T_{e} \times n \times \sqrt{k_{B} T_{e} / m_{i}}$

with $\gamma$ the power sheath transmission factor, $\Gamma$ the local particle flux density, and $m_{i}$ the mass of the ions of the SOL plasma (here the deuterium mass $m_{D}=2 \times m_{p}$ is taken). The effect of screening of the particle from plasma heating by a cloud of evaporated material is neglected. Introducing the exponential SOL profiles the convective heating power becomes

$$
\begin{aligned}
P_{C}= & 2 O_{\perp} \times \gamma \times n(L C F S) / 4 \times\left[k_{B} T_{e}(L C F S)\right]^{3 / 2} \times \\
& \exp \left[-\left(d_{S O L}-x\right) / \lambda_{n}\right] \times \exp \left[-3 / 2\left(d_{S O L}-x\right) / \lambda_{T_{e}}\right] .
\end{aligned}
$$

For the hot particle investigated here the sheath transmission factor has to include the effects of thermal as well as secondary electron emission (SEE) and a possible space-charge limitation of the respected currents. 


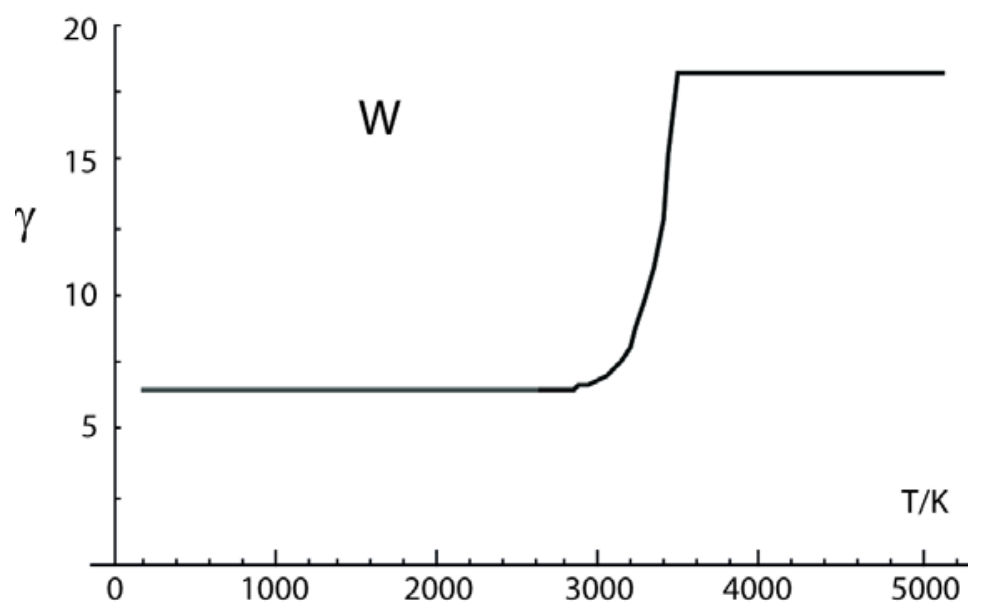

Figure 6. Energy sheath transmission factor for the deposition of power from the SOL-plasma onto the flying $\mathrm{W}$-particle.

\subsection{The sheath transmission factor}

At the melting temperatures of $\mathrm{W}$ the material is principally at emissive conditions. The calculation of the sheath transmission factor $\gamma$ follows [11]. Thermal emission described by the Richardson formula as well as secondary emission are taken into account. For the integration over the distribution of electron energies in the plasma a Maxwellian is assumed. The space-charge limitation causes a lower bound of $\mathrm{V}_{\mathrm{s}} \geq 0.7 \times \mathrm{k}_{\mathrm{B}} \mathrm{T}_{\mathrm{e}}$ as well as an upper bound $\mathrm{j}_{\mathrm{E}} \geq 5 \times 10^{6} \mathrm{~A} / \mathrm{m}^{2}$ of the current density from thermal emission and, therefore, defines a maximum value for $\gamma(\approx 18.9$ in the case of $\mathrm{W})$. The result is shown in figure 6 .

\subsection{The mass balance}

Due to evaporation the macro-particle looses mass at the rate $\mathrm{r}$. The typical thermal velocity $\mathrm{v}_{\text {th }}$ of $\mathrm{W}$ -

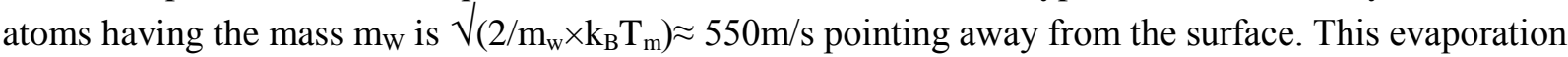
produces a cloud of $\mathrm{W}$-atoms surrounding the particle. A systematic ionization of the vapor atoms by the SOL-plasma will take place defining a typical ionization length $l_{i}$ along the path of the ejected atom. The created $\mathrm{W}$-ion starts to gyrate with the radius $\mathrm{r}_{\mathrm{G}}=\mathrm{Ze} \times \mathrm{v}_{\mathrm{th}, \perp} /\left(\mathrm{m}_{\mathrm{W}} \times \mathrm{B}\right)$ where $\mathrm{v}_{\mathrm{th}, \perp}$ is the component of the thermal velocity perpendicular to the magnetic field. Together with the component $\mathrm{v}_{\mathrm{th}, \|}$ parallel to the field it has to add up to the vector of the original emission velocity.

\subsection{The re-deposition of $W$-ions}

In principle the ion has a chance to hit the surface of the macro-particle again along its gyro-path but the probability is difficult to estimate. For the full thermal velocity the gyro-radius of a singly ionized Wion becomes $\approx 385 \mu \mathrm{m}$, very large as compared with the diameter of a typical macro-particle making it 
difficult for the ion to strike the particle again if the atom was emitted close to perpendicular to the Bfield direction. In the complementary case of an emission almost parallel to the field lines the gyroradius can be comparable with the diameter of the target, but now the parallel component pointing generally away from the particle has to be large. Consequently, a small probability for the re-deposition of an evaporated and ionized $\mathrm{W}$-atom is expected when the particle is small (as compared with the gyro-radius for the thermal velocity). To check the arguments a simulation of the flight of numerous emitted and ionized $\mathrm{W}$-atoms was carried out counting the re-deposition events. The result shown in figure 7 demonstrates that the contribution of re-deposited $\mathrm{W}$-ions onto the macro-particle can be neglected in the mass balance.

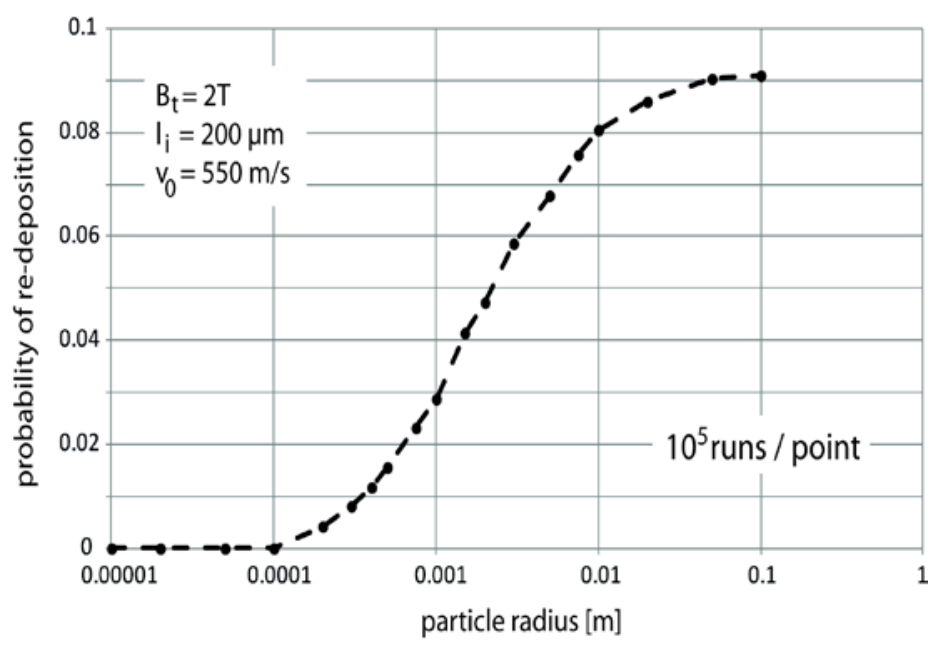

Figure 7. Probability for the re-deposition of a W-ion originating from a macro-particle onto the same particle.

\subsection{The evolution of particle mass and temperature}

Because changes of the momentum of the macro-particle are neglected the time variable in the equations for the mass loss and the temperature rise can be replaced by the depth $\mathrm{x}$ into the SOL using the constant velocity $\mathrm{v}_{0, \perp}$. The system of coupled equations reads then

$\mathrm{d} m(x) / \mathrm{d} x=-r(T) \times O_{p} / v_{0, \perp}$

and

$\mathrm{d} T / \mathrm{d} x=\left(P_{c}-P_{r}-P_{e v}\right) /\left(m \times c_{W}\right) \times 1 / v_{0, \perp}$

with $c_{W}$ the specific heat capacity of the particle's material. The mass $m_{0}=m(x=0)$ calculated for the volume of a sphere with the diameter $\mathrm{d}(\mathrm{x}=0)$ and the melting temperature $\mathrm{T}_{\mathrm{m}}$ for $\mathrm{W}$ are used as the initial conditions (at $\mathrm{x}=0$ ) for the two variables $\mathrm{m}(\mathrm{x})$ and $\mathrm{T}(\mathrm{x})$, respectively,

$m(x=0)=m_{0}=\pi / 6 \times d^{3}(x=0) \times \rho$

and

$T(x=0)=T_{m}$.

The equations are integrated numerically until $\mathrm{x}=\mathrm{d}_{\mathrm{SOL}}$ or the position where the mass $\mathrm{m}(\mathrm{x})$ vanishes. As results either the location $\mathrm{x}_{\mathrm{ev}}$ for the complete evaporation of the particle or the ratio $\mathrm{m}_{\mathrm{LCFS}} / \mathrm{m}_{0}$ between the remaining mass passing the LCFS and the starting mass are calculated. 
The profiles of the parameters of the SOL-plasma are determined by $\mathrm{k}_{\mathrm{B}} \mathrm{T}_{\mathrm{e}}(\mathrm{LCFS})=50 \mathrm{eV}$, $\mathrm{n}(\mathrm{LCFS})=1 \times 10^{20} \mathrm{~m}^{-3}$ and the respective decay lengths $\lambda_{\mathrm{Te}}=3 \mathrm{~cm}$ and $\lambda_{\mathrm{n}}=1 \mathrm{~cm}$ (typical for an existing $\mathrm{SOL}$ ). Free parameters are the emission velocity $\mathrm{v}_{0, \perp}$ and the diameter $\mathrm{d}$ of the emitted particle.

\section{Results of the simulation}

Flying from the far out wall into the rather cold and thin SOL plasma the liquid particle evaporates only few material and cools by this evaporation as well as due to radiation (figure 8). At the lower particle temperature evaporation and radiation come to a stop and the particle continuous its flight with almost constant mass and temperature. Approaching the hot and dense part of the SOL-plasma the particle is convectively heated and starts to evaporate and radiate again.

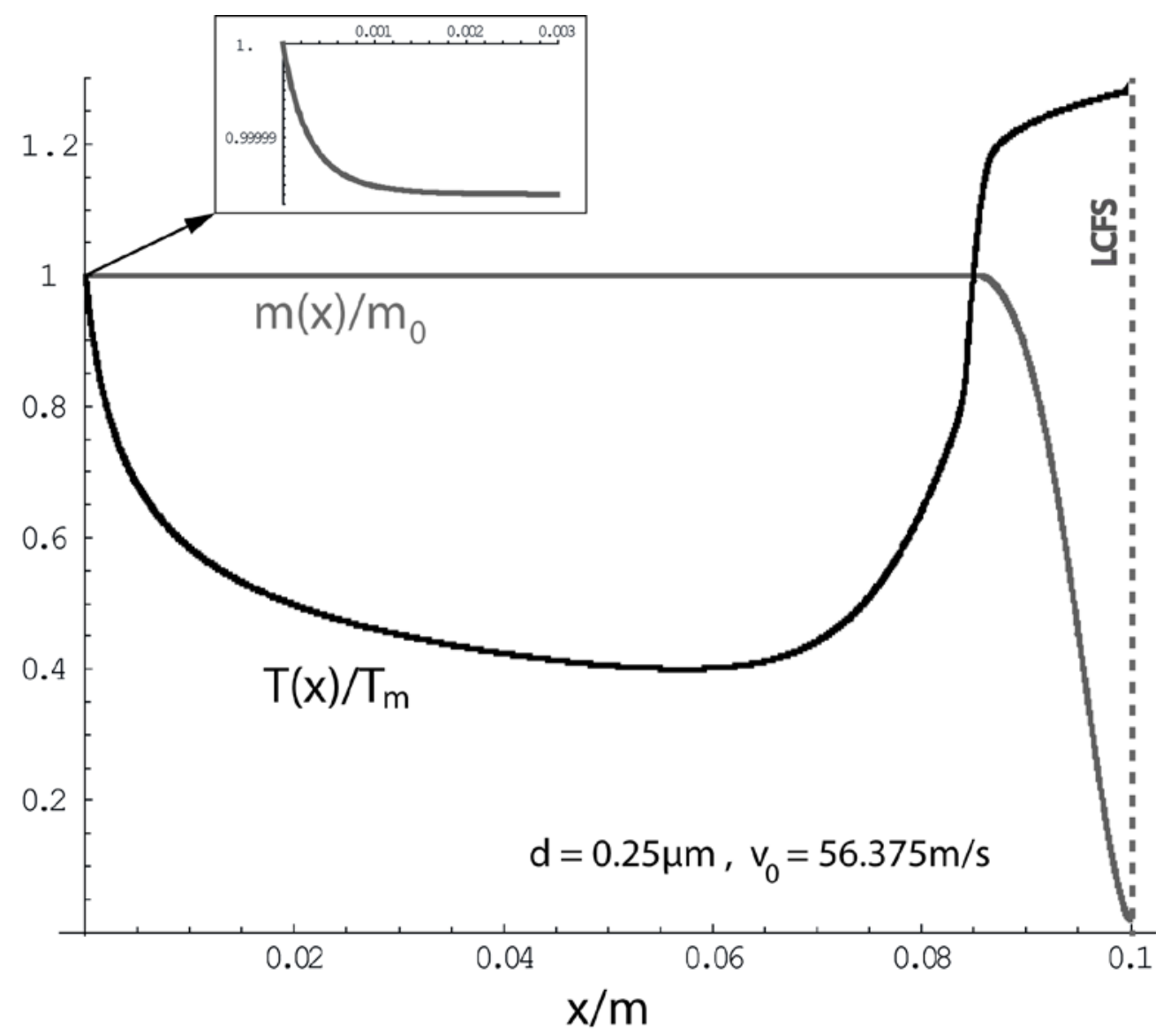

Figure 8. Body temperature and actual mass of a $\mathrm{W}$-particle (starting with $\mathrm{d}=0.25 \mu \mathrm{m}$ and $\mathrm{v}_{0}=56.375 \mathrm{~m} / \mathrm{s}$ ) during its flight through the SOL (n(LCFS) $=10^{20} \mathrm{~m}^{-3}, \mathrm{k}_{\mathrm{B}} \mathrm{T}_{\mathrm{e}}(\mathrm{LCFS})=50 \mathrm{eV}, \lambda_{\mathrm{n}}=10 \mathrm{~mm}$, $\lambda_{\mathrm{T}}=30 \mathrm{~mm}$ ) as a function of the position in the SOL (the inlet shows the evaporation directly after the ejection).

In the segment of the path close to the LCFS the heating and the radiative and evaporation cooling are of the same order of magnitude. If a particle with given start-diameter $d$ vanishes completely or passes the LCFS with its residual mass depends on its ejection velocity $\mathrm{v}_{0, \perp}$. A critical velocity $\mathrm{v}_{0 \text {,crit }}$ is defined by a path where the particle finally evaporates exactly at the LCFS. Systematic simulation scans reveal that depends on $d$ as $v_{\text {crit, } \perp}=D_{2} / d$. For velocities $v_{0, \perp}>v_{0, \text { crit }}$ the particle crosses the LCFS with the mass $\mathrm{m}$ (LCFS) that is entering the region of closed flux surfaces (see figure 9). This amount m(LCFS) was found to depend on the velocity $\mathrm{v}_{0, \perp}$ and the particle's starting diameter $\mathrm{d}$ approximately as

$m(L C F S)=\pi / 6 \times d^{3} \times\left\{1-\exp \left[-\left(v_{0, \perp} \times d-D_{2}\right) / D_{1}\right]\right\} \times \rho$

where the abbreviations $D_{1} \approx 56 \mu \mathrm{m} \times \mathrm{m} / \mathrm{s}$ and $\mathrm{D}_{2} \approx 13.9 \mu \mathrm{m} \times \mathrm{m} / \mathrm{s}$ are introduced (both valid for the prescribed set of SOL parameters). 
The critical velocity $\mathrm{v}_{0, \text { crit }}$ depends on the parameters of the SOL-plasma in the expected way: larger $\mathrm{n}$ or $T_{e}$ at the LCFS call for larger velocities to reach the LCFS and longer decay lengths stretch the heating time and, therefore, also result in a larger $\mathrm{v}_{0, \text { crit }}$.

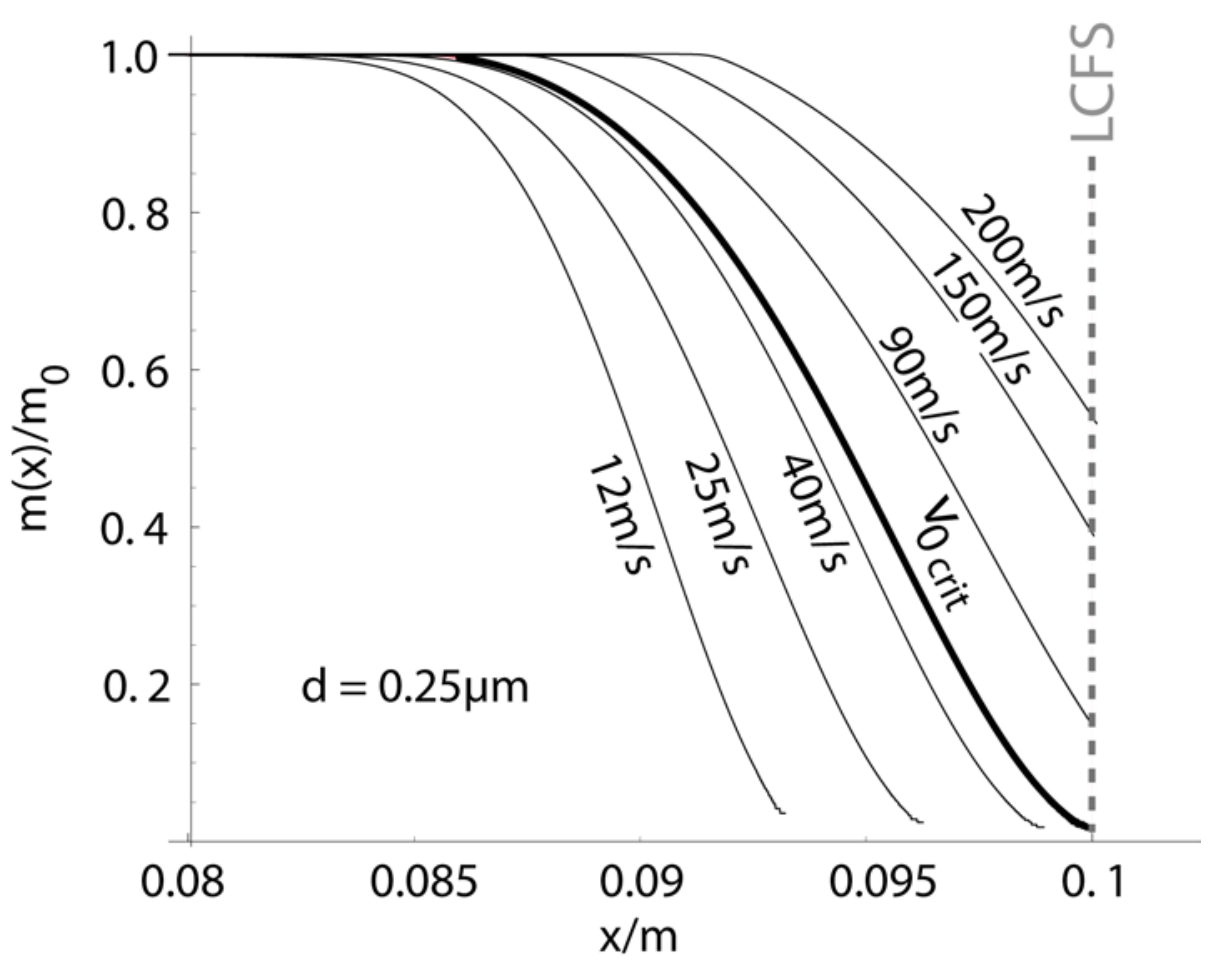

Figure 9. Ratio of the mass remaining at the LCFS to the mass at ejection depending on the position in the SOL.

\section{Results and conclusions}

Macro-particles emitted from cathode-spots of arcs burning on W-coated PFC's inject W into the SOL circumventing the mechanism of prompt re-deposition. Hence they are one among several sources of $\mathrm{W}$ in the machine.

The particles start at melting temperatures and, therefore, evaporate little material close to the PFC. Due to radiative cooling along the path they solidify until the plasma heating melts them again and $\mathrm{W}$ material is liberated approaching the LCFS. In most cases the particle will be completely evaporated in the SOL so that only a part of the released W can be transported into the main plasma. Occasionally, depending on the perpendicular velocity and the starting mass of the particle, a solid relic may pass the LCFS thereby injecting $\mathrm{W}$ directly into the confined region. For every diameter $\mathrm{d}$ of an particle a minimum velocity for passing the LCFS is given by $v_{\text {crit }, \perp}=D_{2} / d$.

Figure 10 shows the number of particles flying with velocities seen in experiment to be larger than the required minimum $v_{\text {crit, },}(\mathrm{d})$. Data are taken from the experimentally observed velocity distribution published in [1] (see figure 15). It can be concluded that to pass the LCFS particles having diameters smaller than $\approx 0.2 \mu \mathrm{m}$ require velocities not seen in experiment. On the other hand particles large enough to pass with velocities experimentally observed are increasingly rare because of the strongly falling diameter distribution ( $\sim \mathrm{d}^{-4.7}$, see fig. 3). Therefore, only a fraction of the particles produced are able to transport a massive remnant across the LCFS. To estimate the amount of material two examples are discussed. 


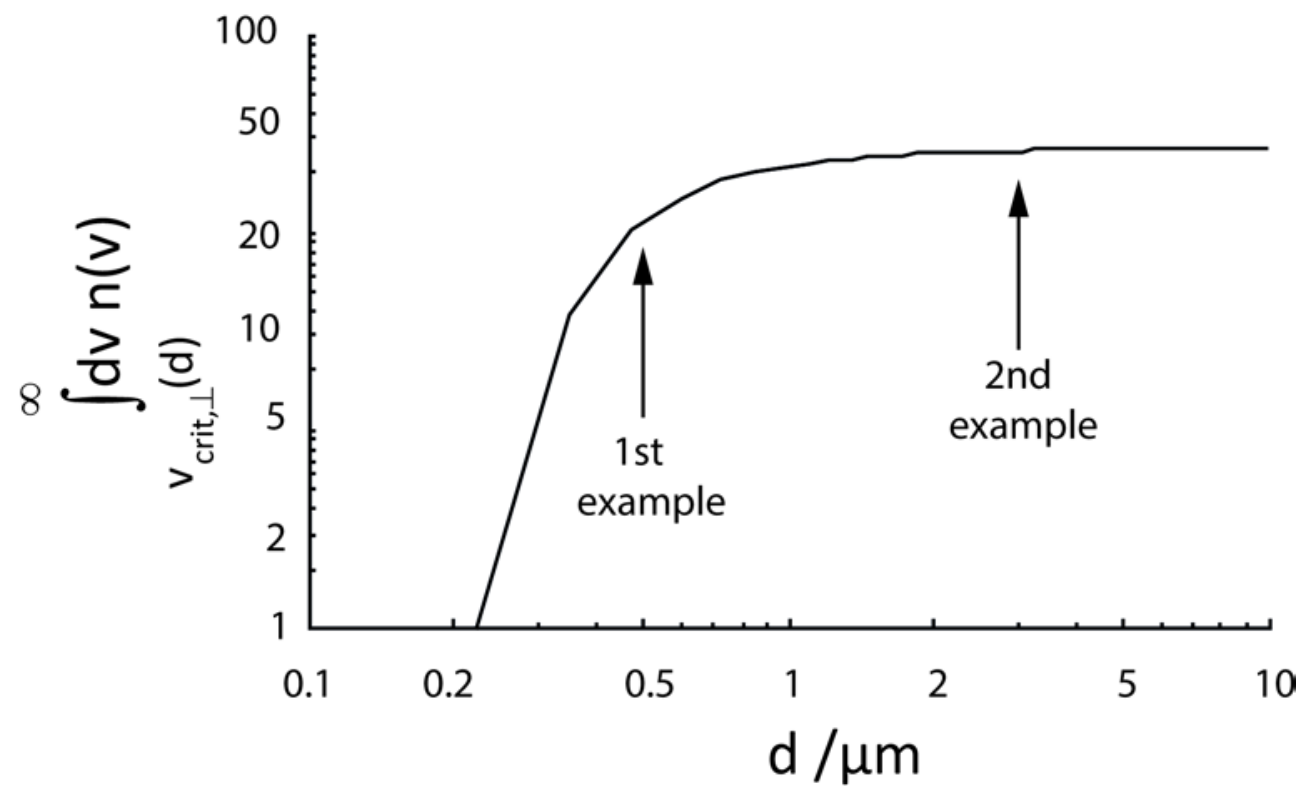

Figure 10. Sum of all particles having velocities (in experiment) larger than $v_{\text {crit }, \perp}(d)$ for a prescribed diameter $\mathrm{d}$. All these particles would inject $\mathrm{W}$ material into the inner plasma if their diameter (not observed in the same experiment) is $>=\mathrm{d}$.

$1^{\text {st }}$ example: One of the numerous typical particles $(\mathrm{d}=0.5 \mu \mathrm{m})$ having a velocity of $35 \mathrm{~m} / \mathrm{s}$ (seen to appear well within the velocity distribution) injects about $8 \times 10^{-14} \mathrm{~g}(6 \%$ of its original mass) or $2.5 \times 10^{10} \mathrm{~W}$ atoms. But to calculate the overall contribution of those particles one has to multiply by the number of particles having $\mathrm{d} \approx 0.5 \mu \mathrm{m}$ and the assumed velocity of $35 \mathrm{~m} / \mathrm{s}$ emitted by one individual arc and, finally, by the number of arcs - both numbers are currently not known.

$2^{\text {nd }}$ example: For a very rare large particle $(d=3 \mu \mathrm{m})$ flying with the average velocity $(20 \mathrm{~m} / \mathrm{s})$ it can be expected that $50 \ldots 60 \%$ of the original mass are injected in a single event. This are $1.5 \times 10^{-10} \mathrm{~g}$ equivalent to $5 \times 10^{13} \mathrm{~W}$ atoms (for comparison the number of D-atoms in a typical ASDEX Upgrade discharge is $10^{21}$ ).

Of course, the projection of the real ejection velocities onto the perpendicular direction narrows the window for particles capable of passing mass over the LCFS. This can be an essential discrimination if (as indicated in figure 4) large particles have a preference for shallower ejection angles. Otherwise the number of arcs burning simultaneously can be large and the number of macro-particles emitted by every active cathode-spot is large as well.

Unfortunately, the distribution of arc tracks over the first wall is not homogeneous and the physics behind it is unclear making a scale-up difficult. Additionally, size, velocity, and direction of an ejected particle are probably not independent parameters. For an estimate of the amount of introduced material and the variation in time and location additional information about the interrelation of the particle parameters is needed (preferably from laboratory experiments).

But still this leaves a probability for a pile-up of $\mathrm{W}$ in the confined plasma produced by either a large number of small contributions distributed over time (example 1) or a few large remnant solid Wparticles (example 2). In the latter case the entering within a critical time for removal (roughly given by the particle confinement time) may result in a discharge with a problematic W impurity content lacking a conceivable reason. 


\section{References}

[1] Laux M, Siemroth P, Marx M, Neu R, Rohde V, Balden M and Endstrasser N 2013 J.Nucl.Mater. 438 S758-S761

[2] Rohde V, Endstrasser N, v. Toussaint U, Balden M, Lunt T, Neu R, Hakola, Bucalossi J and ASDEX Upgrade Team 2011 J. Nucl. Mater. 415 S46-S50

[3] Boxman R L and Goldsmith S 1981 J.Appl.Phys. 52 151-161

[4] Krasheninnikov S I, Smirnov R D and Rudakov D L 2011Plasma Phys. Control. Fusion 53 1-54

[5] Schülke T and Anders A 1999 Plasma Sources Sci. Technol. 8 567-571

[6] Anders 1997 J.Appl.Phys. 82 3679-3688

[7] Krauz V I, Martynenko Yu V, Svechnikov N Yu, Smirnov V P, Stankevich V G and Khimchenko L N 2010 Physics-Uspekhi 53 1015-1038

[8] Naujoks D et al. 1996 Nucl. Fusion 36 671-687

[9] Boxman R L, Sanders D M and Martin P J (Eds.) 1995 Handbook of Vacuum Arcs Science and Technology, Material Science and Process Technology Series, Noyes Publications, Park Ridge, New Jersey, USA,

[10] Espe W 1959 Werkstoffkunde der Hochvakuumtechnik, Bd. 1 44-45 VEB Verlag der Wissenschaften, Berlin

[11] Tokar M Z, Nedospasov A V and Yarochkin A V 1992 Nucl. Fusion 32 15-24 